\title{
Expression diversity and evolutionary dynamics of rice duplicate genes
}

\author{
Won Cheol Yim $\cdot$ Byung-Moo Lee $\cdot$ Cheol Seong Jang
}

Published online: 17 March 2009

(C) Springer-Verlag 2009

\section{Erratum to: Mol Genet Genomics}

DOI 10.1007/s00438-009-0425-y

Author would like to make single correction in his abstract part of Online published article. In the right column of first page 7th line instead of 'more than 60\%' it should be read as 'more than $\mathbf{3 0 \%}$ '.

The online version of the original article can be found under doi: 10.1007/s00438-009-0425-y.

W. C. Yim · B.-M. Lee

Department of Plant Biotechnology,

Dongguk University, Seoul 100-715, South Korea

C. S. Jang $(\bowtie)$

Plant Genomics Lab, Division of Bio-resources Technology,

Kangwon National University, Chuncheon 200-701, South Korea

e-mail: csjang@kangwon.ac.kr 